\title{
Stronger quantum advantages with photons
}

\author{
Xiaosong Ma \\ Nanjing University, School of Physics, Nanjing, China
}

Quantum computing ${ }^{1}$ promises exponential speed-up in for such tasks as factorizing, simulating many-body quantum systems, and quantum machine learning. However, to achieve such computational speed-up is far from trivial. A universal quantum computer needs at least: (1) many qubits that can be individually coherently controlled; (2) many quantum gates/operations that can establish correlations among many qubits; and (3) fault-tolerance architecture that can correct the errors during computation, and hence output the correct answers. Each of these three requirements is highly demanding to realize.

In 2012, John Preskill proposed a novel concept, "quantum supremacy," also known as the "quantum advantage" (we use the latter term throughout this commentary), which defines the goal of demonstrating that a quantum device can solve a problem that no classical computer can solve efficiently. Boson sampling ${ }^{3}$ is one such problem, in which the outcome statistics of bosons emerging from a large and complex circuit is difficult to simulate on classical computers. Photon, as a boson, is the natural candidate for realizing such a task, because the required multiparticle quantum interference can be naturally implemented thanks to its bosonic nature. ${ }^{4}$

The recent experiment, Jiuzhang 2.0 (Fig. 1), ${ }^{5}$ by Jian-Wei Pan and Chao-Yang Lu's group has significantly improved experimental demonstration over the landmark experiment reported last year by the same group. ${ }^{6}$ The improvements are three-fold. Firstly, by using stimulated emission of two-mode squeezed states, the detected photon number increases from 76 to 113 . Note that every increased photon expands the experimental accessible Hilbert space exponentially. So, the additional 37 photons is a huge deal. Secondly, the whole experiment is programmable by reconfiguring the phases of the input states. This brings the Boson sampling machine ${ }^{7}$ one-step closer to the computer we have in mind, in which we can program the machine and obtain input-dependent results. Thirdly, the scale of the photonic circuit grows from 100 to 144-mode. By combining all three improvements, they obtain a Hilbert space dimension up to $\sim 10^{43}$, and a sampling rate $\sim 10^{24}$ faster than using brute-force simulation on classical supercomputers. This is a remarkable achievement in the era of quantum advantage.

Since the first quantum advantage experiment demonstrated by the Google team in 2019, the race between quantum systems and classical algorithmic simulations never stops, much like the increasingly complicated quantum Bell experiments that continue to refute classical local hidden variable theories, as discussed in Ref. 5. Experimentally, increasing the quality and mode number of the quantum machine with more photons will allow further increase in the dimensionality of the Hilbert space, which will make the classical algorithmic simulations increasingly difficult, and hence reach an even stronger quantum

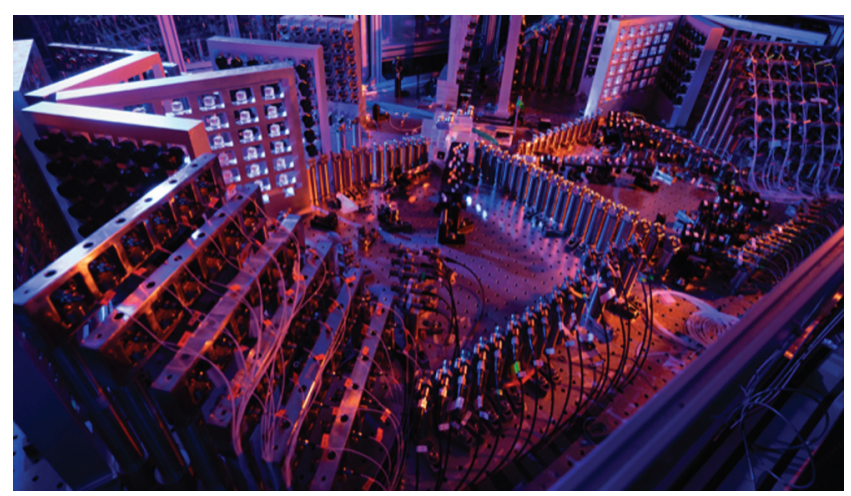

Fig. 1 Jiuzhang 2.0 experiment uses a 144-mode interferometer to obtain a sampling rate $\sim 10^{24}$ faster than using brute-force simulation on classical supercomputers. Credit: Chaoyang Lu, University of Science and Technology of China.

advantage for a problem that is intractable with classical computation power.

\section{References}

1. T. D. Ladd et al., "Quantum computers," Nature 464, 45-53 (2010).

2. A. W. Harrow and A. Montanaro, "Quantum computational supremacy," Nature 549, 203-209 (2017).

3. S. Aaronson and A. Arkhipov, in Proc. Forty-Third Annual ACM Symp. on Theory of Computing., Association for Computing Machinery, pp. 333-480 (2011).

4. D. J. Brod et al., "Photonic implementation of boson sampling: a review," Adv. Photon. 1(3), 034001 (2019).

5. H.-S. Zhong et al., "Phase-programmable Gaussian boson sampling using stimulated squeezed light," Phys. Rev. Lett. 127(18), 180502 (2021).

6. H.-S. Zhong et al., "Quantum computational advantage using photons," Science 370(6523), 1460-1463 (2020).

7. C. S. Hamilton et al., "Gaussian boson sampling," Phys. Rev. Lett. 119(17), 170501 (2017).

Xiaosong $\mathrm{Ma}$ is a professor in the School of Physics at Nanjing University. He obtained his BS degree from Nanjing University in 2003, his MS degree from Leiden University in 2005, and his PhD from University of Vienna in 2010. A subsequent postdoctoral research post in experimental quantum physics at University of Vienna, was followed by a postdoc in integrated quantum photonics at Yale University. 